\title{
Traditional Healing - At What Cost ?: A Case Report from Rural India
}

\author{
Vishalkumar Shah ${ }^{1}$, Ajinkya Sureshrao Ghogare ${ }^{2}$, Swaroopa Vasant Lungepatil ${ }^{3}$, Ganpatlal \\ Kodarbhai Vankar ${ }^{4}$ \\ ${ }^{1,2}$ Assistant Professor, Department of Psychiatry, Datta Meghe Institute of Medical Sciences, Sawangi \\ ${ }^{3}$ Junior Resident, Datta Meghe Institute of Medical Sciences, Sawangi \\ ${ }^{4}$ Professor, People's College of Medical Sciences and Research Centre, People's University, Bhopal \\ Corresponding author: Ajinkya Ghogare \\ Email - ajinkyaghogaremd@gmail.com
}

\begin{abstract}
In rural parts of India still the "magico-religious beliefs" regarding the occurrence of the psychiatric disorders and "magico-religious treatments" of the psychiatric disorders often seen and prevail. Such beliefs and treatments many times lead to physical as well as psychological trauma to an individual suffering from any psychiatric disorder and may lead to delay in seeking an appropriate professional help in time. The present case report attempts to depict what kind of physical and psychological trauma a person with psychiatric disorder suffered at the hands of faith healer in absence of lack of awareness about the psychiatric disorders among family members and other villagers.
\end{abstract}

Key words: Psychiatric disorders, faith healer, magico-religious beliefs, magico-religious treatments, rural India, physical trauma, psychological trauma.

(Paper received $-10^{\text {th }}$ October 2019 , Peer review completed $-19^{\text {th }}$ October 2019 )

(Accepted $-25^{\text {th }}$ October 2019)

\section{INTRODUCTION}

According to the International Classification of Diseases, Tenth revision (ICD - 10), acute and transient psychotic disorder is characterized by an acute onset of delusions, hallucinations, incomprehensible or incoherent speech, or any combination of these symptoms. The time interval between the emergence of the symptoms for the first time and the presentation of fully formed disorder should not exceed two weeks. The disorder doesn't meet the criteria for manic episode, depressive episodes or recurrent depressive disorder. The disorder doesn't occur in the course of any organic condition, any other mental or medical conditions including substance dependence, intoxication, harmful use or substance withdrawal state. Acute and transient psychotic disorder may or may not be associated with acute stress. [1-2].

Psychiatric disorders in India are often attributed to be caused by supernatural phenomena and many times patients are subjected to different types of "magico-religious treatments" by faith healers [3]. The term "magico-religious" refers to a cultural belief in supernatural ideas, such as sorcery, spirits, divine intervention, astrology, and reincarnation. The term "magico-religious beliefs" refers to all supernatural influences believed a specific culture. The treatment by indigenous healers, religious people is collectively called as, "magico-religious treatment" [4]. The healers engage in violent practices like beating the patient, whip, force to walk on nailed shoes, throw stone, lighting camphor on bare hands and asking to swallow it, restraining with chains/ ropes, fumes of burning chilies, to name a few. They also employ not so violent but disagreeable measures like tonsure of head, tying sacred thread., prophesize by indigenous healer, applying kajal over betel leaf to predict future, rituals wearing neem leaves bunch as dress to worship god, rubbing the 
lemon on head directly in order to bring down pita, sprinkling/spraying holy water during prayer, dipping tantric plate in to water, pooja rituals in graveyard [4].

We are reporting here the case of 26 years old married woman with acute transient and psychotic disorder who lost her eyesight following the violent treatment of her psychiatric disorder from a faith healer.

\section{CASE REPORT}

Index patient is a 26 years old Hindu married female belonging to a nuclear family, educated up to tenth standard and working as a farmer. She got married two years back. But after marriage she had alleged conflicts with her husband over the issue of household work. According to husband patient was less educated than him and she was not performing household duties properly. In the two years span of their marriage, patient's husband brought her to her maternal home on four occasions. Six months back following another verbal conflict, he dropped her to her maternal house and warned her that he is no more interested in continuing the relationship with her. Two months back patient had filed a case against her husband. Fifteen days back while returning back from farm with her mother, patient suddenly started muttering to self, using abusive language, shouting against her husband as well as people from maternal home and removing her body cloths. Mother took her to home with the help of villagers. Whenever family members tried to calm her down she would start shouting at them by using abusive language and beating them with her bare hands. Then she started complaining against some unknown people that they had done black magic on her and were causing family conflicts. She remained awake throughout the night and kept on talking to herself loudly disturbing other family members. This continued for eight days. On advice of few villagers, patient's parents thought that their daughter was possessed by some evil spirit and decided to take help of local faith healer who was known to remove the evil spirits and set that person free of evil influence.

Parents brought that faith healer to their house to treat their daughter. Faith healer asked the parents to give him lemons so that he can mix them with the special medicine he was having and calling his special medicine as "Lep". On receiving the lemons, faith healer asked the parents to wait outside. After that parents heard shouting voices of the patient. Patient was tied at that moment. After the completion of procedure in about one hour, parents entered the house and they were shocked to see the condition of their daughter's eyes. Her both eyes were severely red in colour, she was having severe burning sensation in both the eyes. She was crying loudly and was having bleeding from both eyes. She was shouting that she can't see anything. Her skin around both eyes and skin over nasal bridge was having burns and it was red in colour.

Parents asked the faith healer what kind of treatment he had subjected to patient, the reply of the faith healer was "just wait for few hours, your daughter will be free of the evil spirit". After that incident because of pain and inability to see anything clearly, patient's symptoms got aggravated and she started shouting more loudly by using abusive language against the family members, faith healer and her husband.

Three days after that incident of treatment from local faith healer, parents brought her to the hospital for treatment. At the time of presentation in the casualty, patient was shouting loudly, using abusive language, talking with herself and blaming her parents as well as faith healer for her condition. On presentation, patient was not able to open her eyelids of left eye, while the eyelids of right eye were partially opened with reduced blinking rate. The skin around her both eyes and over nasal bridge was white in colour. Eyelashes were burnt and intermingled with each other. She was admitted under the combined care of department of Psychiatry and department of Ophthalmology. She was started on Tablet Olanzapine $10 \mathrm{mg}$ at bed time and Tablet Clonazepam $0.5 \mathrm{mg}$ at bed time. Senior Ophthalmologist have started her on antibiotic eye drops to take care of infections and injuries. After onset of treatment, she became calm and began to deny delusions, fearfulness or hearing of voices not heard by others. She became more concerned about her eyesight and started crying intermittently, fearing total and permanent loss of her eyesight. She kept on asking her parents and doctors whether she will regain her eyesight or not. Later on, she was able to count the fingers up to two feet distance only. She was not able to see the faces clearly and was telling the examiner that she can only perceive black and white images of the person standing and sitting near her. She was not able to read and write. Her ophthalmological examination revealed that she had "corneal epithelial damage with corneal 
scarring". Her right eye cornea was nearly white in colour while on the left side both the eyelids were closed and it was not possible to open the left eye. Patient was referred to higher centre for the purpose of corneal transplantation.

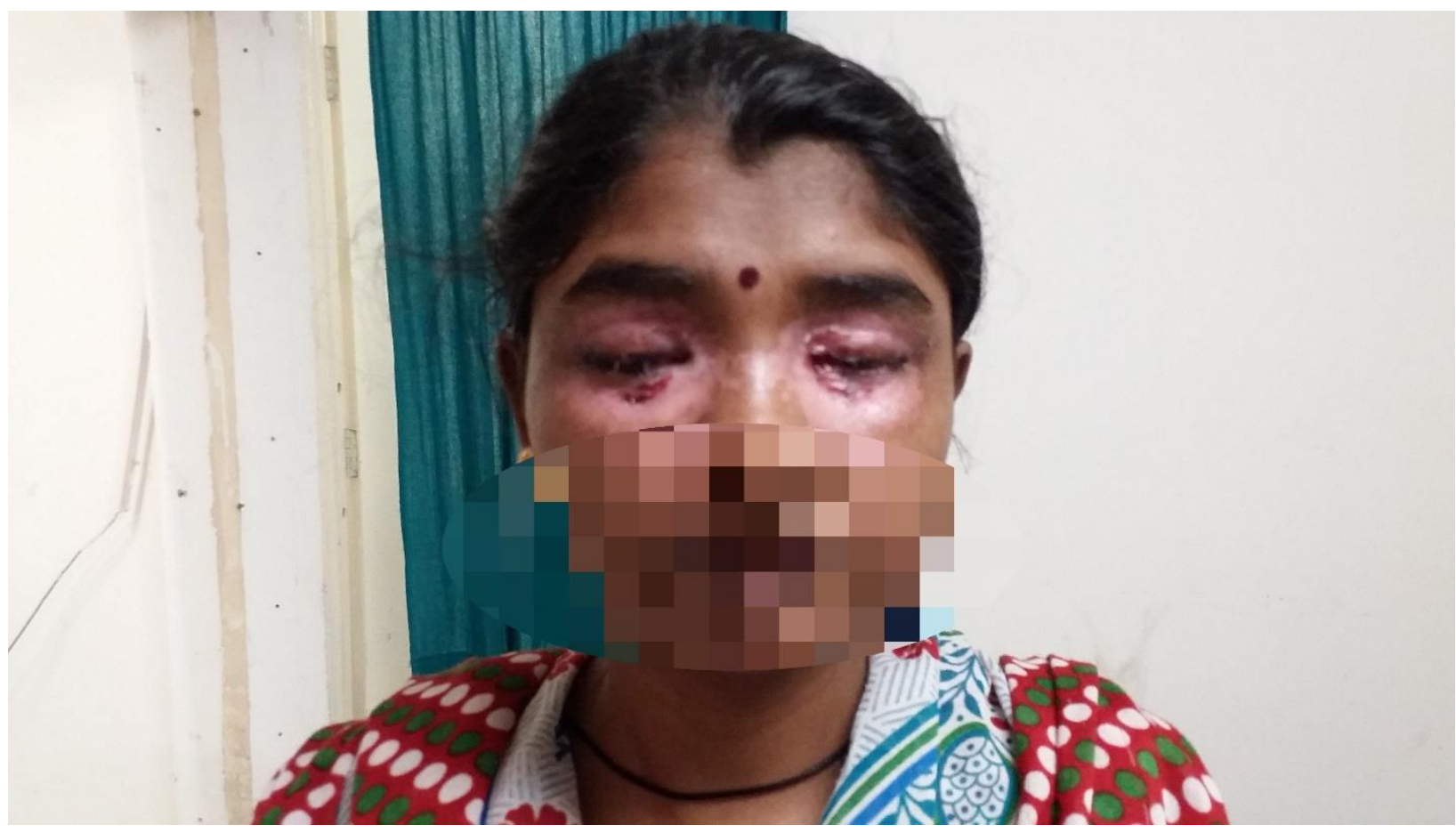

Figure 1: White discoloration of the skin around both eyes and over the nasal bridge

\section{DISCUSSION}

The case presented showed lack of awareness among the family members as well as villagers regarding the psychiatric disorders. Kulhara and others observed that many people from Northern India believe that supernatural phenomena play significant role in causation of psychiatric disorders and such beliefs tend to subject persons suffering from any psychiatric disorders to "magico-religious treatments"[3]. Kar observed that in case of psychiatric disorders, faith healers are the predominant choice for the majority of the population because $75 \%$ of the patients take recourse to folk or religious healing before resorting to psychiatric services [5]. Magico-religious beliefs have long been believed to cause psychiatric disorders not only in India, but in other countries well. Vlachos observed that in 80 mothers of psychotic patients, $85 \%$ entertained ideas related to metaphysical and magico-religious causation of psychotic disorders in their children. Resorting to exorcism or magic was very common among them [4]. Velayudham and others observed that a majority $(64 \%)$ of caregivers and neighbours believed in black magic and majority (56\%) attributed psychiatric disorders to black magic. The belief in black magic is central to the problem of people seeking faith healers [4]. They also observed that $56 \%$ of care givers believed that God's anger was the causative factor in occurrence of psychiatric disorders. These kinds of beliefs also promote seeking faith healers as primary source of contact [4]. Joel and others observed that $87.5 \%$ of the study participants from southern India attributed origins of psychiatric disorders to non-biomedical causes, such as black magic and evil spirits [6]. Popat-Jain observed that cultural beliefs had a negative relationship with attitudes toward treatment, demonstrated that individuals who held more culturally based beliefs, had a more negative attitude toward seeking treatment [7]. Shidhaye and Vankar explored traditional healing practices in 201 psychiatric patients attending Psychiatry OPD in a tertiary care centre in Western India. 54.7\% patients in this study irrespective of diagnosis consulted a traditional healer. Sixteen of the 110 patients had to pay an amount greater than Rs 2500 . The view that traditional healing is effective for mental health problems was supported by $22.7 \%$ of the patients and $20.9 \%$ would recommend going to a traditional healer [8]. 


\section{CONCLUSION}

Magico-religious beliefs are common in rural population of India. Many caregivers and patients attribute the symptoms of psychiatric disorders to magico-religious beliefs. Such beliefs lead to increase duration of untreated psychiatric disorders and delays appropriate treatment seeking in psychiatric settings. Not only that the faith healer engages in torturing rituals which may damage patient's body and its functioning, sometimes permanently. Thus, there is need to increase the awareness among the population from rural India about psychiatric disorders and available treatment modalities like pharmacotherapies and psychotherapies.

\section{Declaration of patient consent}

The authors certify that they have obtained the signed consent form from patient's parents as patient was not able to sign the consent form because of eye injury. Parental signature was obtained after taking the permission from patient. In the consent form, patient and her parents have given the consent for the image and other clinical information to be reported in the journal. The patient and the parents understand that name and initials will not be published, and due efforts will be made to conceal identity, but anonymity cannot be guaranteed.

\section{REFERENCES}

1. World Health Organization. International statistical classification of diseases and related health problems: instruction manual. World Health Organization; 2004.

2. World Health Organization. The ICD-10 classification of mental and behavioural disorders: clinical descriptions and diagnostic guidelines. Geneva: World Health Organization; 1992.

3. Kulhara P, Avasthi A, Sharma A. Magico-religious beliefs in schizophrenia: A study from North India. Psychopathology 2000;33(2):62-8.

4. Vlachos IO, Beratis S, Hartocollis P. Magico-religious beliefs and psychosis. Psychopathology 1997;30(2):939.

5. Kar N. Resort to faith-healing practices in the pathway to care for mental illness: a study on psychiatric inpatients in Orissa. Mental Health, Religion and Culture 2008;11(7):720-40.

6. Joel D, Sathyaseelan M, Jayakaran R, Vijayakumar C, Muthurathnam S, Jacob KS. Explanatory models of psychosis among community health workers in South India. Acta Psychiatr Scand 2003;108(1):66-9.

7. Popat-Jain AR. Beliefs about Mental Illness and Its Impact on Attitudes toward Treatment: An Examination of the Asian-Indian Immigrant Population in the United States (Doctoral dissertation, Northeastern University); 2011.

8. Ningsangrenla L, Rao PS. Traditional Healing Practices and Perspectives of Mental Health in Nagaland. J North East India Stud 2019;9(2):33-56.

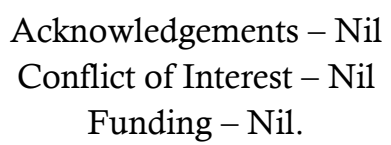

